\title{
Open-Source Low-Cost Antenna Measurement Setup for Rapid Echoic Measurements
}

This paper was downloaded from TechRxiv (https://www.techrxiv.org).

\section{LICENSE}

CC BY 4.0

SUBMISSION DATE / POSTED DATE

$13-09-2021 / 16-09-2021$

\section{CITATION}

Wagih, Mahmoud; Moody, Thomas (2021): Open-Source Low-Cost Antenna Measurement Setup for Rapid Echoic Measurements. TechRxiv. Preprint. https://doi.org/10.36227/techrxiv.16610818.v1

$\mathrm{DOI}$ 


\title{
Open-Source Low-Cost Antenna Measurement Setup for Rapid Echoic Measurements
}

\author{
Mahmoud Wagih, Member, IEEE, and Thomas Moody \\ School of Electronics and Computer Science, University of Southampton, SO17 1BJ, United Kingdom \\ orcid.org/0000-0002-7806-4333
}

\begin{abstract}
With the massive growth in wireless systems and the internet of things vision, low-cost and rapid antenna measurements in echoic lab environments have attracted significant research, commercial, and educational interest in the last decade. In this paper, we present a low-cost antenna positioning system based on open-source positioning hardware, for in-situ 3-dimensional radiation pattern measurements. The positioner is an off-the-shelf Arduino robotic arm and can perform azimuth and elevation measurements over a hemisphere using different rotation mechanisms. The positioning system is used in conjunction with a commercial Vector Network Analyzer (VNA) and wire $\lambda / 2$ antennas for the second VNA port. The measured radiation patterns of a flexible microstrip patch antenna using the proposed setup are compared to 3D fullwave simulations as well as anechoic chamber measurements. The measurement setup, excluding the VNA, costs less than $\$ 300$, and does not require calibrated reference antennas, rotary connectors, or specific PC peripherals. The open-source design library is publicly available at git.soton.ac.uk/mahm1m19/ antennameasurement_system/.
\end{abstract}

Index Terms - antenna radiation patterns, antenna measurements, open-source, radiation patterns

\section{INTRODUCTION}

With industry reports expecting a trillion wirelessly connected devices by 2035, an interest surge in antenna development and testing activities is anticipated. The ubiquity and rapid development life-cycles of such Internet of Things (IoT) devices implies a need for rapid and low-cost insitu antenna measurement setups. In addition, low-cost and scalable hands-on antenna teaching approaches will be of paramount importance for training antenna designers for a reliably-connected IoT.

Commercial Vector Network Analyzers (VNAs) have gone down in cost to less than $\$ 1,000$. Moreover, several highlyintegrated VNAs have been demonstrated for rapid and lowcost testing applications, showing similar performance to high-end test instruments [1]. Nevertheless, low-cost and rapid antenna radiation pattern measurements remain a challenge [2]. Commercially available systems for rapid and low-cost antenna measurements are now available from RF instrumentation vendors such as Megiq [3], and Dreamcatcher [4], aimed towards rapid lab-based measurements and education environments. However, the cost of such solutions, despite being lower than their higher-load higher-accuracy counterpart, is still high, e.g. $>\$ 15,000$ for the Megiq system [3] while still requiring an external RF signal source.

Several studies described low-cost antenna pattern measurement systems [5]-[9]. In [5], an in-house "open-source"

This work was supported by the UK Engineering and Physical Sciences Research Council (EPSRC) under Grant EP/P010164/1 positioning system was utilized in conjunction with software defined radios for a low-cost setup, validated in an echoic environment with a single absorbing surface to reduce reflections [5]. Nevertheless, the details of such setup were not made publicly available. Other implementations included the use of an automated azimuth rotary sage with manual elevation rotation [6]. When combined with a time-gated high-end VNA, the co-polarized measured patterns exhibited a very close agreement with $3 \mathrm{D}$ simulation for a broadside patch antenna [6]. However, cross-polarization levels were not reported, and the use of the VNA's time-gating function restricts the setup to high-end VNAs with time-domain option. More recently, multiple low-cost systems have been demonstrated as part of the IEEE Antennas \& Propagation Symposium student competition [7]-[9]. Yet, most of these systems were not evaluated for complete spherical pattern measurements [7], or are limited to hemispherical measurements at a single frequency [8]. Furthermore, the design details, such as the firmware and automation scripts, are not publicly available which hinders simple replication of the developed setups.

In addition to works dedicated to realizing low-cost rapid antenna measurement setups, several antenna design studies have utilized in-house manual or automated pattern measurement techniques in echoic environments [10]. Low-cost antennas, such as thin flexible RF energy harvesting rectennas [10] and textile-based positioning antennas [11] were evaluated using indoor measurements outside an anechoic range. For both the omnidiriectional [10] and directional antennas [11], a satisfactory agreement between the simulated and measured patterns was demonstrated.

In this paper, we detail an open-source 3D antenna radiation pattern measurement setup, based on an open-source off-the-shelf (COTS) Arduino-controlled robotic arm positioner, a commercial VNA, and a PC-run Matlab script. The proposed setup, capable of automated hemispheric pattern measurements, is evaluated in an indoor environment showing its suitability for rapid antenna testing and demonstration purposes, which are expected to attract significant interest for future wireless devices. The system's components are described in Section II with the experimental validation in Section III; the open-source repository containing the developed software is accessible from [12].

\section{SySTEM DESIGN AND IMPLEMENTATION}

To realize an automated positioner for spherical radiation pattern measurements, both elevation and azimuthal rotations need to be incorporated in the PC-controllable system. The 


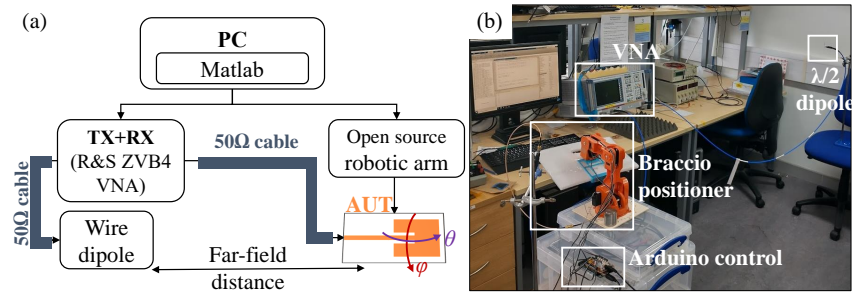

Fig. 1. The proposed low-cost open-source radiation patterns measuremen setup: (a) block diagram; (b) photograph in an echoic environment.
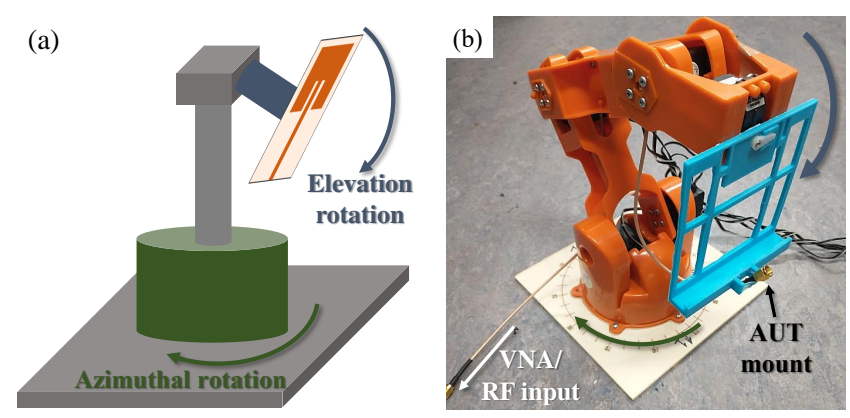

Fig. 2. Layout (a) and photograph (b) of the Braccio positioner showing the rotation axes and the AUT mount.

proposed system uses an open-source robotic arm (Braccio) which can be easily controlled using an Arduino firmware over several rotational axes. The Braccio was previously explored as a single-axis antenna positioner, showing minimal influence on a dipole's radiation patterns when used in an anechoic range [13]. On the control PC, a Matlab script communicates with the Arduino via a serial USB bus. The same script communicates with the RF measurements hardware, in this case a Rohde \& Schwarz ZVB4 VNA, over a crossover ethernet connection. By avoiding the GPIB interface not found in most PCs, the costs of the test setup can be further reduced eliminating the need for costly IEEE 488.2 adapters or peripherals. The system's block diagram is shown in Fig. 1(a).

The implemented rotation mechanism of the Braccio robotic arm is shown in Fig. 2. Azimuthal XY rotation is achieved using the servo controlling the base-plate of the Braccio. By utilizing lightweight flexible SMA-SMA cables, both the coaxial RF input and the servo's power and control wires can be routed within the robot's body. The system has an approximate load capacity of 500 gm for the antennaunder-test (AUT), limited by the Braccio's servos.

Unlike most elevation positioners, the developed setup tilts the robotic arm holding the AUT, as illustrated in Fig. 2(a), to rotate the AUT over the elevation plane. While such tilting mechanism $=$ varies the position of the antenna relative to the transmitter, which may reduce the measurement accuracy, it eliminates the need for expensive rotary connections, as well as improves the mechanical reliability of the positioner and the stability of the mounted AUT. Furthermore, as evaluated in the next section, it is found that the tilting positioner results in comparable accuracy to its counterparts in literature which operate based on standard axial rotations [5].

To measure the co and cross-polarized components of the antenna's patterns, the Matlab script, [12], prompts the user to change the polarization of the source prior to resuming the automated scanning by the Braccio arm. This could be achieved using manual connections to a dual-polarized antenna such as a quad-ridged horn, by physically rotating a linarely-polarized antenna, or by electrically-controlled RF switches synchronized using the same automation script.

Due to the limited $0-180^{\circ}$ rotation range of the Braccio's servos, the setup was designed to perform automated hemispherical measurements. The user is prompted to manually rotate the positioner by $180^{\circ}$ over the XY azimuth plane to measure the second hemisphere of the antenna's radiation patterns. This limitation can be addressed by utilizing a different servo capable of rotating the AUT by $360^{\circ}$ over the XY plane.

\section{System EVAluAtion}

The system was evaluated in an indoor echoic lab environment, shown in Fig. 1(b). The positioner was placed at $1.5 \mathrm{~m}$ from the second port antenna. The "reference" antenna used in this work was a simple $\lambda / 2$ wire dipole soldered onto a low-cost SMA connector to eliminate the need for directional antennas such as quad-ridged horns or gain-standard log periodic antennas required in previously reported setups [3], [6]. However, the use of a more directional source will reduce the multi-path reflections off the surrounding surfaces which can further improve the measurement repeatability and accuracy. A video demonstration of the setup in operation is available through the Git Wiki [12].

The radiation patterns of the AUTs measured using the proposed setup are compared to simulated radiation patterns from CST Microwave Studio. Electromagnetic simulation has been widely used to validate the performance of low-cost antenna measurement setups [6], [14]. Polarized spherical patterns were measured with an angular step size of $5^{\circ}$ over both the azimuthal and elevation planes. The co/cross polarization measurements were performed by rotating the measurement dipole antenna $90^{\circ}$. As detailed in the design section, the semi-automated measurements were performed as a four-step process as follows:

1) Measure the co-polarized radiation over hemisphere 1;

2) Rotate the Braccio by $180^{\circ}$ to measure hemisphere 2;

3) Reset the Braccio position and rotate the polarization of the source to measure the cross-polarized hemisphere 1;

4) Rotate the Braccio by $180^{\circ}$ to measure the crosspolarized radiation over hemisphere 2 .

First of all, a proximity-fed broadside microstrip patch antenna implemented on a low-cost felt substrate, designed to operate at the first-order TM mode at $2.4 \mathrm{GHz}$, was used as the AUT. Fig. 3 shows the CST-simulated and Bracciomeasured 3D radiation patterns of the antenna. Observing both plots, it can be observed that the broadside radiation of the patch is clearly observed with minimal differences over the antenna's main-lobe. Over the second hemisphere, i.e. the back radiation into the patch's ground, it can be observed that the measured patterns exhibit higher radiation and subsequently a lower front-to-back ratio compared to simulations. This is greatly attributed to the multi-path effects 

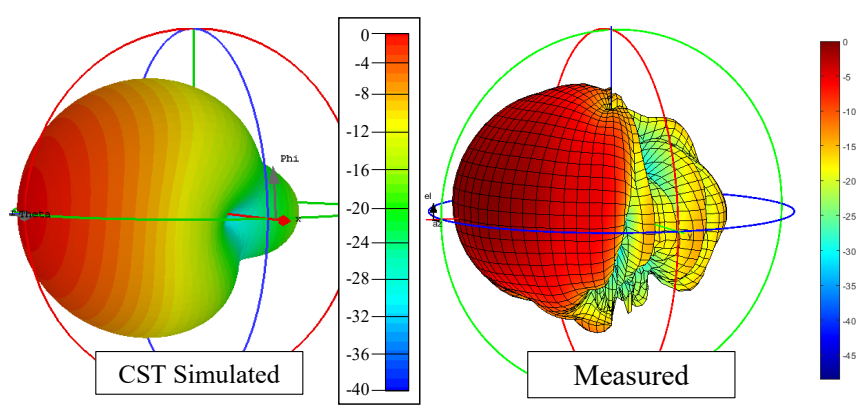

Fig. 3. CST-simulated and measured 3D relative gain patterns of a proximity-fed microstrip patch operating at $2.4 \mathrm{GHz}$.

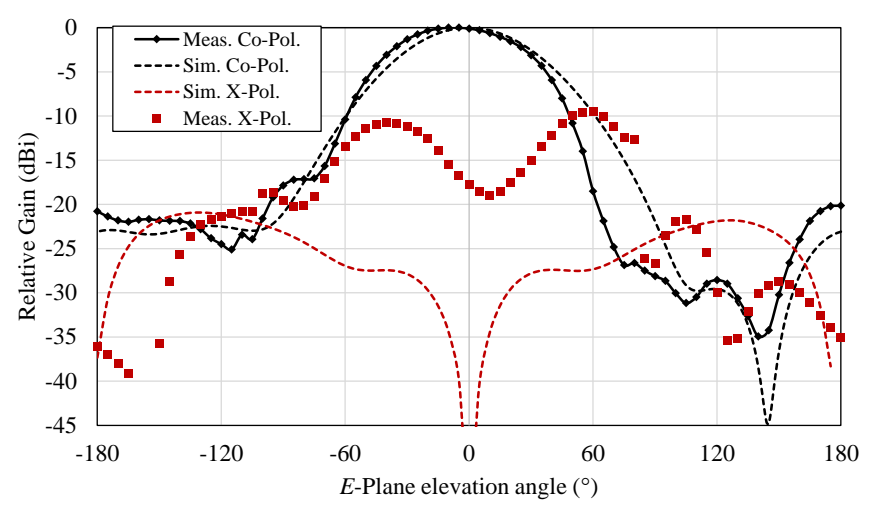

Fig. 4. Comparison of the CST-simulated and measured 2D gain patterns of the same proximity-fed microstrip patch operating at $2.4 \mathrm{GHz}$ showing the co and cross-polarization levels.

in the indoor environment, making it difficult to detect radiation "nulls".

To further investigate the discrepancies introduced by the low-cost echoic setup, the 2D E-plane elevation patterns are shown over a Cartesian plot in Fig. 4. Observing the co-polarized patterns, it can be shown that while both simulated and measured main-beams exhibit a resemblance, the AUT's measured patterns appear to be more directive than the simulated patterns. This can be attributed to the reflections off the positioner. Similar beam-narrowing effects were reported when a similar microstrip patch was measured using a commercial positioner in an anechoic chamber but in presence of a human body phantom [15].

As for the back-radiation, it can be seen that both the simulated and measured pattern exhibit good agreement with a front-to-back ratio of at least $20 \mathrm{~dB}$. Therefore, front-toback ratios between 0 and $20 \mathrm{~dB}$ could be resolved using the proposed low-cost positioner in an echoic environment. The cross-polarized patterns however show a significant discrepancy of over $10 \mathrm{~dB}$ in the main-lobe direction. Not only is the higher measured cross-polarized component due to reflections off the positioner, but also due to polarization impurities arising from the omnidirectional source. To explain, the multi-path effects introduced by the dipole's omnidirectional patterns will result in a higher cross-polarized channel gain between the AUT and the measurement antenna. Therefore, using a high-discrimination dual-polarized antenna will further improve the co/cross-polarization accuracy of the measurements. It was previously found that a low-

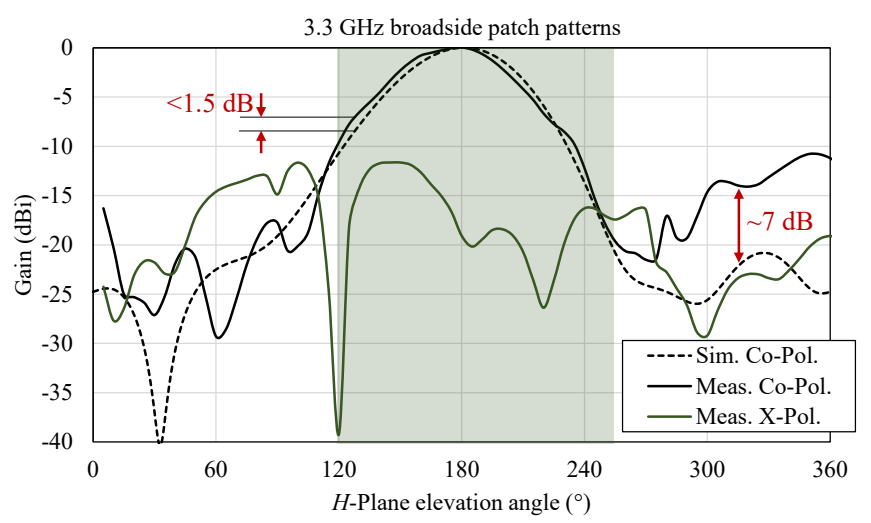

Fig. 5. CST-simulated and measured co and cross-polarized patterns of a $3.3 \mathrm{GHz}$ microstrip patch; the highlighted region indicates the angles at which the discrepancy is under $1.5 \mathrm{~dB}$.

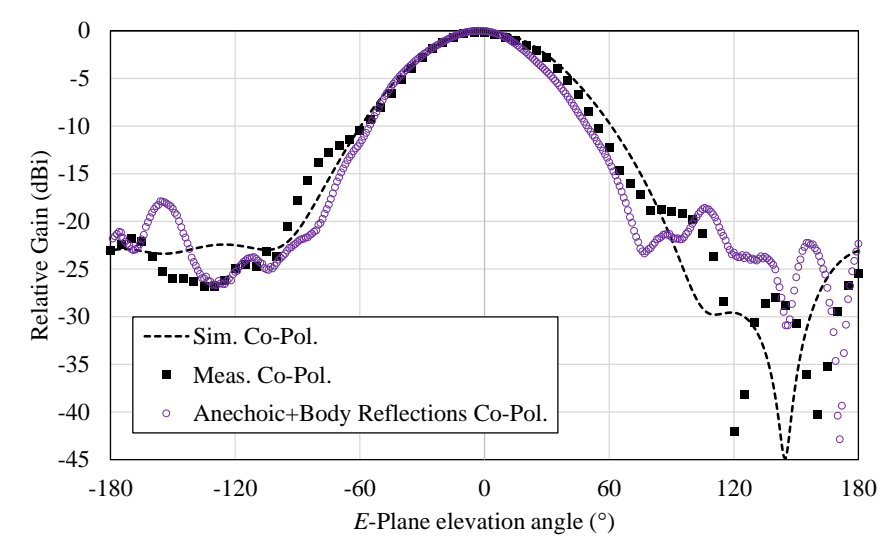

Fig. 6. Comparison of the measured echoic patterns of a $2.4 \mathrm{GHz}$ proximitycoupled patch with anechoic chamber measurements in [16].

cost single-axis turntable realized uinsg Lego and a stepper motor produced low cross-polarization measurements (under $-20 \mathrm{dBi}$ ) when used inside an anechoic chamber [9].

To demonstrate the frequency scalability of the developed setup, a $3.3 \mathrm{GHz}$ microstrip patch was also measured. Fig. 5 shows the simulated and measured polarized patterns of the $3.3 \mathrm{GHz}$ patch's $H$-plane. While the simulated and measured main lobe patterns appear to be in very good agreement with less than $1.5 \mathrm{~dB}$ discrepancy, the measured back radiation and front-to-back ratio exhibit higher discrepancies compared to the $2.4 \mathrm{GHz}$ patch in Fig. 4. This can be moslty attributed to additional reflections off the surroundings. In particular, it can be observed that the discrepancy increases to 7$10 \mathrm{~dB}$ due to the placement of the VNA and the control PC approximately $90-180^{\circ}$ off the patch's $H$-plane centre. Therefore, the highest accuracy measurements can only be achieved in a large space with off-ground and off-wall clearances larger than the separation between the AUT and the measurement dipole. The cross-polarized patterns for the 3.3 $\mathrm{GHz}$ patch were also found to be significantly higher than their simulated level, not shown in Fig. 4 due to being under $-40 \mathrm{dBi}$.

In addition to the CST-based validation of the developed setups, the radiation patterns of a broadside patch antenna are compared to those measured on a human body phantom in an anechoic chamber using a commercial positioning 
system [16]. While the anechoic measurements eliminate the reflections off the surroundings, the antenna was mounted on a radiation absorbing body phantom to mimic realworld shadowing effects. The simulated, anechoic chambermeasured, and low-cost setup-measured patterns are shown in Fig. 6.

Observing the echoic and anechoic measurements from -60 to $60^{\circ}$, it can be seen that the measured patterns using the developed low-cost setup and the commercial positioner in the anechoic chamber are in very good agreement with under $1 \mathrm{~dB}$ variations. The radiation null observed around $140^{\circ}$ in simulation is found to result in different responses in both the anechoic and echoic measurements, highlighting the challenge in characterizing the patterns precisely under $-30 \mathrm{dBi}$. Nevertheless, the $>25 \mathrm{dBi}$ radiation patterns are in good agreement.

\section{Conclusion}

In this paper, we presented a low-cost open-source antenna radiation pattern measurement setup based on a PCcontrolled COTS robotic arm and a standard VNA. Despite the very low cost nature of the system, it can be concluded that echoic measurements can provide a sufficient level of accuracy for rapid characterization of future IoT antennas, and for education environments where portability and short test time are key. The proposed setup was characterized for antenna measurements at $2.4 \mathrm{GHz}$ and $3.3 \mathrm{GHz}$ showing reasonable accuracy in the main-lobe direction of a lowcost microstrip patch antenna with around $1.5 \mathrm{~dB}$ deviation from the antennas' simulated patterns. Future work includes further improvements of the positioner's rotation mechanism to improve the repeatability of the results as well as to minimze reflections off the robotic arm. The limited use of absorbing sheets and small-scale pyramidal absorbers can also be investigated to reduce the reflections along with an investigation of the optimal operation environment. The data and code underlying the developed system are available from an actively updated git repository at https://git.soton.ac.uk/ mahm1m19/antennameasurement_system [12].

\section{ACKNOWLEDGEMENT}

The authors would like to thank Dr. Geoffrey S. Hilton from the University of Bristol Communications Systems and Networks group for providing the measured radiation pattern of the patch antenna from [16].

\section{REFERENCES}

[1] H. Chung, Q. Ma, M. Sayginer, and G. M. Rebeiz, "A packaged 0.01-26-ghz single-chip sige reflectometer for two-port vector network analyzers," IEEE Transactions on Microwave Theory and Techniques, vol. 68, no. 5, pp. 1794-1808, 2020.

[2] B. Dewandaru, F. Y. Zulkifli, and E. T. Rahardjo, "A simple radiation pattern measurement for large array antennas," in 2019 IEEE Conference on Antenna Measurements Applications (CAMA), 2019, pp. $325-328$.

[3] "370MHz to $4 \mathrm{GHz}$ Radiation Measurement System," Megiq, Online: https://www.megiq.com/products/rms-0440. Accessed 09/2021.

[4] "ME1300 Antenna and Propagation Courseware," Dreamcatcher, 2009, Online: https://www.avantec2.cl/imagenes/pdf/Catalogo ME1300.pdf. Accessed 09/2021.

[5] C. W. Hearn, D. S. Birch, D. Newton, and S. L. Chatlin, "Open-source antenna pattern measurement system," in 2020 Antenna Measurement Techniques Association Symposium (AMTA), 2020, pp. 1-6.
[6] A. Rehman, T. Rasul, M. F. Shafique, B. Ijaz, K. S. Alimgeer, M. S Khan, R. M. Shubair, and N. K. Mallat, "Development of a cost effective antenna radiation pattern measurement setup," in 2016 16th Mediterranean Microwave Symposium (MMS), 2016, pp. 1-4.

[7] L. Leitao, M. Neves, F. Pinto, G. Maniezo, T. Ferreira, J. N. Matos, and A. Rocha, "2019 ieee ap-s student design contest - team knowantenna [education corner]," IEEE Antennas and Propagation Magazine, vol. 62, no. 5, pp. 120-125, 2020.

[8] Y. Abdalla, M. Wasfy, S. Elmeadawy, N. Abdelhadi, and H. F Hammad, "An antenna characterization demo system using an rfid hemispherical dome [education corner]," IEEE Antennas and Propagation Magazine, vol. 62, no. 3, pp. 107-123, 2020.

[9] A. Gupta, J. Argyres, R. L. Gesner, D. R. Heileman, D. M. Feaster, C. G. Christodoulou, and F. Ayoub, "Diy antenna studio: A costeffective tool for antenna analysis [education corner]," IEEE Antennas and Propagation Magazine, vol. 63, no. 2, pp. 83-88, 2021.

[10] M. Wagih, N. Hillier, S. Yong, A. S. Weddell, and S. Beeby, "Rfpowered wearable energy harvesting and storage module based on etextile coplanar waveguide rectenna and supercapacitor," IEEE Open Journal of Antennas and Propagation, vol. 2, pp. 302 - 314, 2021.

[11] A. Dierck, H. Rogier, and F. Declercq, "A Wearable Active Antenna for Global Positioning System and Satellite Phone," IEEE Trans. Antennas Propag., vol. 61, no. 2, pp. 532 - 538, 2013.

[12] M. Wagih and T. Moody, "Antenna Measurement System," Git, 2021, Online: https://git.soton.ac.uk/mahm1m19/antennameasurement_ system/-/wikis/home. Accessed 09/2021.

[13] A. Knowles, "Radiated Antenna Pattern Measurement on a budget. Phase 1." 2017, Online: https://radtenna.com/blog/ radiated-antenna-pattern-measurement-on-a-budget-phase- 1 .

[14] C. W. Hearn, "Open-source antenna pattern validation using feko," in 2020 International Applied Computational Electromagnetics Society Symposium (ACES), 2020, pp. 1-2.

[15] M. Wagih, G. S. Hilton, A. S. Weddell, and S. Beeby, "Dual-polarized wearable antenna/rectenna for full-duplex and mimo simultaneous wireless information and power transfer (swipt)," IEEE Open Journal of Antennas and Propagation, vol. 2, pp. 844-857, 2021.

[16] - "2.4 ghz wearable textile antenna/rectenna for simultaneous information and power transfer," in 2021 15th European Conference on Antennas and Propagation (EuCAP), 2021, pp. 1-5. 